\title{
A SAFT-VR+DE equation of state based approach for the study of mixed dipolar solvent electrolytes
}

\author{
Gaurav Das, ${ }^{\mathrm{a}}$ Stepan Hlushak, ${ }^{\mathrm{a}}$ and Clare $\mathrm{M}^{\mathrm{c}} \mathrm{Cabe}^{\mathrm{a}, \mathrm{b}}$ \\ ${ }^{a}$ Department of Chemical and Biomolecular Engineering, and \\ ${ }^{b}$ Department of Chemistry, Vanderbilt University, Nashville, TN 37235, United States
}

\begin{abstract}
The SAFT-VR+DE approach was developed by combining the statistical associating fluid theory for potentials of variable range (SAFT-VR) with integral equation theory and the generalized mean spherical approximation using a non-primitive model, in order to explicitly describe the solvent in electrolyte solutions. Subsequently, the theory was applied to study nineteen different aqueous electrolyte solutions and a range of thermodynamic properties across different temperatures and salt concentrations were successfully predicted. Here, the theory is applied to study several model mixed solvent electrolytes in order to validate a simple one-fluid-like approximation that is proposed in order to describe the interactions between ions and dipolar solvents of arbitrary size and dipole moment. Before application to real fluids the approach is extensively tested through comparison with isothermal-isobaric ensemble (NPT) Monte Carlo simulations for a number of model mixed solvent electrolyte systems. Simulations have been performed for different ionic concentrations, solvent compositions, cation, anion, and solvent segment diameters, and solvent polarity. The results show that the SAFT-VR+DE equation provides a good description of the PVT behavior of the model mixed solvent electrolyte systems studied, with some over-prediction of the PVT behavior observed at higher ionic concentrations. Additionally, comparisons are made between predictions from the non-primitive and primitive models for electrolytes and the importance of capturing the effect of the ions on the solvent dielectric demonstrated.
\end{abstract}

Keywords: ions, dipoles, electrostatics, molecular model, theory 


\section{Introduction}

Mixed solvent electrolyte solutions are of significant industrial interest due to their application in several chemical processes such as wastewater treatment, crystallization, liquid-liquid extraction, extractive distillation, and seawater desalination [1]; however, only limited experimental data on the thermodynamic properties of these fluids compared to aqueous electrolyte systems are available [2]. An attractive approach to explore such systems is therefore to develop theoretical tools that can predict the phase behavior and thermodynamic properties of mixed solvent electrolytes as a means of generating "pseudo" experimental data. Though this is made more difficult by the fact that these fluids are highly non-ideal systems because of the dominant long-range electrostatic interactions, which makes their theoretical study challenging.

Theoretical developments for electrolyte solutions can be broadly divided into two approaches: those in which the solvent is described implicitly by a dielectric constant (known as primitive models) [3] and those in which the ions and solvent species are both treated explicitly (known as non-primitive models). The primitive model is a McMillanMayer level of theory [4], developed with the notion that an explicit representation of the solvent is not necessary and can be treated implicitly within the theory. This approximation is likely to be valid at low ionic concentrations. In contrast, the nonprimitive model allows the explicit description of solvent species within the theoretical framework and is in the general class of Born-Oppenheimer models [4]. In the simplest Born-Oppenheimer models of electrolytes, the electrolyte solution is described as a mixture of charged hard spheres in a solvent of spherical hard dipolar molecules. We note that to date, only the primitive model has been applied to the study of mixed solvent 
electrolytes.

In the context of the development of theoretical approaches for the calculation of the thermodynamic properties of mixed solvent electrolytes, the primitive-model-based semi-empirical Debye-Hückel (DH) theory is the simplest. The DH theory, in which ions are treated as point charges in a dielectric media, is in general perhaps the most widely adopted approach for the treatment of electrostatic interactions because of its simplicity. However, the applicability of DH theory is limited to the very dilute regime of electrolyte solutions. Since its advent the most significant contribution to improving the $\mathrm{DH}$ approach was made by Pitzer. In the Pitzer-Debye-Hückel (PDH) [5] formalism the short ranged effect of electrostatics were accounted for by including ionic strength dependence in the second virial coefficient and thus the DH theory became applicable to higher ionic concentrations. Subsequently a number of theoretical approaches for mixed solvent electrolyte solutions have been developed that combine DH or PDH theory with activity coefficient models such as NRTL, UNIFAC, and UNIQUAC to describe the short ranged dispersive interactions [6-13]. These approaches correspond to the so-called LewisRandall [14] framework in which temperature, pressure and mole fraction of all species are independent variables. In other studies [9, 11], DH/ PDH theory and activity coefficient models, have been further combined with second virial coefficient ${ }^{8}$ or Born contributions in order to provide an improved theoretical representation of the interactions present within mixed solvent electrolytes.

In general, the approaches for mixed solvent electrolytes discussed above typically work as correlative tools with very little or no predictive ability. Furthermore, we note that in some cases there lies a significant inconsistency in the proposed 
theoretical framework $[6,11]$. For example, in the work of Liu et al. [11], the NRTL model is derived from the Lewis-Randall framework, while DH theory is derived from the McMillan-Mayer framework in which the independent variables are temperature, volume, mole fraction of solute and the chemical potential of the solvents. For simplicity the rigorous conversion required between the Lewis-Randall and McMillan-Mayer frameworks are typically ignored $[9,11,15]$; however, the inconsistent treatment of the solvent chemical potential (i.e., as an independent variable in McMillan-Mayer framework and a dependent variable in the Lewis-Randall framework) gives rise to thermodynamic inconsistencies and introduces errors [16]. In order to rectify this inconsistency, several authors [8-10, 16-19] have developed an extended DH formalism that is suitable for use in the Lewis-Randall framework.

A more comprehensive approach to the development of equations of state can be adopted through the use of classical statistical mechanics, which relates intermolecular forces to the thermodynamic functions of fluids. In this regard statistical-mechanicsbased perturbation theories and integral equation theory have both been applied to derive analytical expressions for the Helmholtz free energy due to charged Columbic interactions. Some integral equations (based on the mean spherical approximation closure) provide the advantage of yielding analytical expressions for both the structure and the thermodynamics of the fluid and have been widely utilized in the development of equations of state. In the context of charged fluids, Waisman and Lebowitz [20] and Blum [21] solved analytically the relation between the direct correlation function and the pair correlation function given by the Ornstein-Zernike equation with mean spherical (MSA) closure for the restricted (i.e., an equimolar mixture of equal-diameter hard 
spheres are assumed) and unrestricted primitive models respectively, to derive analytical expressions for Columbic contribution to the thermodynamic properties and Helmholtz free energy. We note that $\mathrm{DH}$ is the limiting case of the primitive model expressions for the MSA i.e., the effect of the volume of the ions is reduced to zero. These analytical expressions of ion-ion interaction free energies were instrumental in the development of statistical associating fluid theory (SAFT) based electrolyte equations of state. SAFT [22$24]$ is a state-of-the-art molecular based equation of state, founded on Wertheim's firstorder thermodynamic perturbation theory (TPT1) [25-27] that in its many variations has been applied to study the thermodynamic properties of a wide range of industrially important complex fluid systems (see for example [28-31]). A MSA term combined with different versions of SAFT has been used by several authors to develop SAFT-based approaches for electrolyte solutions. For example, the SAFT-VRE [32, 33] equation was developed by combining the SAFT approach for potentials of variable attractive range (SAFT-VR) [34] with the MSA expressions for the restrictive primitive model (i.e., an equimolar mixture of equal-diameter hard spheres in a dielectric continuum). In related work, Tan et al. [35] proposed a version of SAFT1 (a variant of SAFT-VR) for electrolyte solutions that also uses the restrictive primitive model. In other examples, the perturbed chain SAFT (PC-SAFT) [36] equation was combined with a simple DH term to develop the ePC-SAFT [33] equation and the polar perturbed chain (PPC)-SAFT equation was combined with the MSA to develop a primitive model of electrolyte solutions in the ePPC-SAFT equation [37]. In these studies we note that the focus was typically on describing the thermodynamic properties and phase equilibrium of single solvent and sometimes mixed salt electrolyte systems; only the SAFT-VRE [32] and 
ePC-SAFT equations have been applied to the study of mixed (i.e., water/ alcohol and water/ alkane) solvent electrolytes.

In the area of mixed solvent electrolyte systems, the SAFT-VRE [32] equation was applied to study the phase behavior of alkane + water + salt solutions [38]. The dielectric constant, required in this primitive model approach, was calculated using a temperature dependent correlation equation obtained by fitting to the pure water experimental dielectric data from 273 to $623 \mathrm{~K}$. The effect of the second solvent or ions on the dielectric behavior of the solution is therefore not accounted for. In the ePC-SAFT equation of state [39], which was applied to study the thermodynamic properties of water + alcohol + salt systems (salts composed of $\mathrm{Na}^{+}, \mathrm{Li}^{+}, \mathrm{K}^{+}, \mathrm{NH}^{+}, \mathrm{Cl}^{-}, \mathrm{Br}^{-}, \mathrm{I}^{-}$ions in ethanol or methanol and water), the solvent composition and temperature dependent dielectric constant data were again obtained from an empirical correlation and required as an input to the equation of state. We note that more recently, a modified version of the SAFTVRE equation [32] in which a Born solvation energy contribution was incorporated into the theory has been proposed and applied to study the VLE/ LLE of four mixed solvent electrolyte solutions. Although the inclusion of the Born term provides a better representation of solvation effects at the infinite dilution limit than in the original SAFTVRE formalism, the explicit representation of long-ranged ion-dipole and dipole-dipole interactions are still absent from the theoretical framework. In this work, the dielectric constant was obtained following the empirical approach of Uematsu and Franck [40], which takes into account the temperature, density and composition of the solvent and in turn makes the dielectric constant differentiable with respect to these same variables. 
A common feature of these SAFT-based approaches and the previously mentioned activity-coefficient-based models is the implicit treatment of the solvent. A more comprehensive approach is to consider solvent molecules explicitly within the electrolyte solution, using a Born-Oppenheimer level model [4]. Analytical expressions to calculate the free energy and thermodynamic properties of a mixture of dipolar and charged hard spheres of arbitrary sizes was derived by Blum and Wei [41] using integral equation theory in which the Ornstein-Zernike equation was solved within the non-primitive MSA (NPMSA) closure. The solution provides mathematical expressions for the contribution to the free energy and pair correlation function for the ion-ion, ion-dipole, and dipoledipole interactions. Recently, Zhao et al. [42] combined the SAFT-VR equation of state and the generalized mean spherical approximation (GMSA) using the non-primitive model to describe the long-range ion-ion, ion-dipole, and dipole-dipole interactions to develop the SAFT-VR+DE equation, a non-primitive-model-based electrolyte version of the SAFT-VR equation. The predictions of PVT behavior for several model electrolyte fluids were compared against NPT Monte Carlo simulation data for systems of different ionic concentrations and different ratio of the cation, anion, and solvent segment diameters to test the accuracy of the approach. Comparisons were also made with the DH and primitive models, illustrating that the full non-primitive model better captures the electrostatic interactions due to the explicit consideration of solvent. The importance of properly accounting for the difference in size between the ions and solvent and providing an accurate description of the dielectric constant was also shown. In subsequent work, the SAFT-VR+DE equation of state was applied to study nineteen aqueous alkali halide electrolyte systems and evaluate a range of thermodynamic properties, including the 
mean ionic activity coefficient, osmotic coefficient, water activity coefficient, density, Gibbs free energy of hydration, and the dielectric decay [43]. The results demonstrate that when developing a predictive equation of state for a family of electrolyte solutions, a uniform model is not appropriate and the nature of the salt and the concentration range being studied should be taken into account and the model tailored to capture the correct interactions. Specifically when studying electrolyte solutions from infinite dilution to the fused salt limit, while a fully dissociated model of electrolytes can be expected to capture the thermodynamic behavior at low to moderate concentrations at higher concentrations, with the changes in intermolecular/ ionic interactions, the underlying theoretical considerations should also change and depending upon the nature of the ions, ion association or enhanced anion-solvent interactions should be considered.

Since statistical-mechanics-based equations of state like SAFT are formulated upon a well-defined molecular model, they have an inherent advantage over more empirical approaches as the approximations made in the development of the theory can be rigorously tested through comparison with computer simulation results for the exact same model. This allows the accuracy of the theory to be determined before comparison with experimental data and parameters are fitted [29]. This approach has been used heavily in the development and testing of new versions of the SAFT equation of state. For example, in the development of the SAFT-VR+D approach, which describes dipolar associating fluids and explicitly considers the effect of dipolar interactions on both the thermodynamics and structure of the fluid, the theoretical framework was extensively tested against isothermal-isobaric (NPT) and Gibbs ensemble Monte Carlo simulation data for the thermodynamic properties and phase behavior of several model dipolar 
square-well monomer and chain fluids before application to experimental fluids. Similarly, as described above the SAFT-VR+DE equation was first validated using computer simulation results [42] before being applied to study experimental systems [43]. Through such studies systematic improvements to the theory can be made that allows the accuracy of the SAFT-based equations to continually evolve.

In this work, the SAFT-VR+DE equation of state has been applied to the study of mixed dipolar solvent electrolyte systems, i.e., ions solvated by at least two distinct dipolar solvent species. Computer simulations have been performed for a series of different molecular models to test the theory before experimental mixed dipolar solvent electrolyte systems are studied. Specifically simulations have been performed in which, the size of the cation, anion, and solvents are assumed to be equal (known as the seminon-primitive model), the ions and solvents are of different sizes while the cation and anion have equal and also different diameters (known as the unrestricted non-primitive model). The effect of the dipole moment of each solvent and ion concentration is also investigated and a comparison with the primitive model of electrolytes included to examine the effect of using a salt-concentration-dependent dielectric constant on the theoretical predictions.

The remainder of the paper is organized as follows: In section 2 we present the SAFT-VR+DE model as applied to mixed dipolar solvent electrolyte solutions, along with the primitive model approach. In section 3, details of the molecular simulations performed are presented. A comparison of the theoretical predictions and simulation results are presented in section 4 and concluding remarks made in section 5 . 


\section{Molecular model and theory}

Mixed dipolar solvent electrolyte solutions are modeled as a mixture of charged ions of arbitrary size within a dipolar associating solvent composed of molecules of arbitrary size and polarity as illustrated in figure 1 . The ions are described as hard spheres, half with charge $+q$ and diameter $\sigma^{+}$, and half with charge $-q$ and diameter $\sigma^{-}$. Two types of dipolar solvent molecules are considered: a monomer fluid that mimics water molecules and a dimer fluid that represents a second solvent such as an alcohol. The monomer solvent is described by diameter $\sigma_{d 1}$ and dipole moment $\mu_{d 1}$ and contains four association sites, two each of different kinds that represent hydrogen bonding sites (i.e., mimicking $\mathrm{H}$ and $\mathrm{O}$ atoms in water). The dimer solvent molecules are described by segments of diameter $\sigma_{d 2}$ and embedded dipole moment of $\mu_{d 2}$ with two association sites.

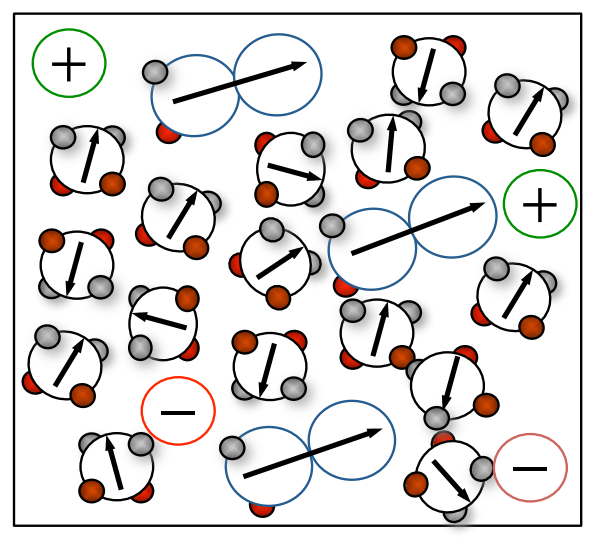

Figure 1. Schematic representation of the molecular model used in the SAFT-VR+DE equation to describe mixed dipolar solvent electrolyte solutions containing ions and two types of associating dipolar solvent. 
The potential model for interaction between solvent and ionic species is given by,

$$
u(r)=u^{S W}(r)+u^{C C}(r)+u^{C D}(r)+u^{D D}(r)
$$

where the square-well, coulombic charge-charge, charge dipole, and dipole-dipole interactions are represented by $u^{S W}(r), u^{C C}(r), u^{C D}(r)$, and $u^{D D}(r)$ potentials, respectively and are defined as follows:

$$
u_{i j}^{S W}(r)=\left\{\begin{array}{cc}
+\infty & r<\sigma_{i j} \\
-\varepsilon_{i j} & \sigma_{i j} \leq r<\lambda_{i j} \sigma_{i j} \\
0 & r>\lambda_{i j} \sigma_{i j}
\end{array}\right.
$$

where the cross interaction square well depth $\left(\varepsilon_{i j}\right)$, range $\left(\lambda_{i j}\right)$ and diameter $\left(\sigma_{i j}\right)$ parameters between molecules $i$ and $j$ are determined using the Lorentz-Berthelot combining rules,

$$
\begin{gathered}
\varepsilon_{i j}=\sqrt{\varepsilon_{i i} \varepsilon_{j j}} \\
\lambda_{i j}=\frac{\lambda_{i i} \sigma_{i i}+\lambda_{i j} \sigma_{i j}}{\sigma_{i i}+\sigma_{i j}}
\end{gathered}
$$

and the arithmetic mean given for the segment diameters

$$
\sigma_{\mathrm{ij}}=\frac{\left(\sigma_{\mathrm{ii}}+\sigma_{\mathrm{jj}}\right)}{2}
$$

The Coulombic charge-charge interaction between ions is given by, 


$$
u_{i j}^{C C}(r)=\left\{\begin{array}{ccc}
+\infty & \text { if } & r \leq \sigma_{i j} \\
\frac{z_{i} z_{j} e^{2}}{4 \pi \varepsilon r} & \text { if } & r>\sigma_{i j}
\end{array}\right.
$$

and the charge-dipole interaction between ions is represented by,

$$
u_{i j}^{C D}(r)=\left\{\begin{array}{ccc}
+\infty & \text { if } & r \leq \sigma_{i j} \\
\frac{z_{i} e \mu}{4 \pi \varepsilon r^{2}}(\hat{r} . \hat{n}) & \text { if } & r>\sigma_{i j}
\end{array}\right.
$$

where $z_{i}$ and $\mu_{i}$ are the charge on ion $i$ and the dipole moment of the $i$ th solvent molecule respectively. The dipole-dipole potential between ions is represented by

$$
u_{i j}^{D D}(r)=\left\{\begin{array}{ccc}
+\infty & \text { if } & r \leq \sigma_{i j} \\
-\frac{\mu^{2}}{4 \pi \varepsilon r^{3}} D\left(\mathbf{n}_{\mathbf{1}} \mathbf{n}_{\mathbf{2}} \hat{\mathbf{r}}\right) & \text { if } & r>\sigma_{i j}
\end{array}\right.
$$

where

$$
D\left(\mathbf{n}_{1} \mathbf{n}_{2} \hat{\mathbf{r}}\right)=3\left(\mathbf{n}_{1} \cdot \hat{\mathbf{r}}\right)\left(\mathbf{n}_{2} \cdot \hat{\mathbf{r}}\right)-\mathbf{n}_{1} \cdot \mathbf{n}_{2}
$$

Here $\hat{r}$ is the unit vector in the direction of $r$ joining the center of the segments and $n_{i}$ is a unit vector parallel to the dipole moment of segment $i$.

The solution of the Ornstein-Zernike with the NPMSA closure proposed by Blum et al. [44] was for ions of arbitrary sizes in a dipolar solvent with a separate segment diameter. No solution of the NPMSA has been developed to date for ions of arbitrary size in a solvent composed of more than one type of dipolar species. This limitation to the application of the NPMSA to the study of mixed dipolar solvent electrolytes has been circumvented in this work by using a one-fluid type approximation in the ion-dipole and dipole-dipole terms within the SAFT-VR+DE equation of state. 
It is therefore assumed that the properties of the solvent can be described by those of a hypothetical or effective fluid that is represented through an effective segment diameter $\left(\sigma_{e f f}\right)$ and dipole moment $\left(\mu_{e f f}\right)$ with parameters determined via [45-47]:

$$
\begin{aligned}
& \sigma_{e f f}^{3}=\sum_{i=1}^{n_{\text {ofleg }}} \sum_{j=1}^{n_{\text {solvent }}} x_{s, i} x_{x, j} \sigma_{i j}^{3} \\
& \mu_{\text {eff }}=\frac{\sum_{i=1}^{n_{\text {polar }} n_{\text {polatr }}} \sum_{j=1} x_{i} x_{j} m_{i} m_{j}\left(\frac{\mu_{i}}{m_{i}} \frac{\mu_{j}}{m_{j}}\right)^{\frac{l}{2}}}{\left(\sum_{i=1}^{n_{\text {polar }}} x_{i} m_{i}\right)^{2}}
\end{aligned}
$$

Even though, an effective fluid has been utilized in this study, the proposed mixing rules in eq. 10-11, implicitly include the effect of solvent composition. Equation 10 represents the van der Waals one fluid mixing rule for size [45], while equation 11 for effective dipole moment is based upon the work of Karakatsani et. al. [47]. These approximations were chosen as they were found to yield better results for the thermodynamic properties of interest compared to other approximations based on the concept of random mixing [48].

To describe the model electrolyte fluids being studied within SAFT-VR+DE theoretical framework the Helmholtz free energy per molecule is given by

$$
\frac{A}{N k_{b} T}=\frac{A^{\text {ideal }}}{N k_{b} T}+\frac{A^{\text {mono }}}{N k_{b} T}+\frac{A^{\text {chain }}}{N k_{b} T}+\frac{A^{a s s o c}}{N k_{b} T}
$$

where $N$ is a total number of molecules, $k_{B}$ is the Boltzmann constant, $T$ is temperature, and $A^{\text {ideal }}, A^{\text {mono }}, A^{\text {chain }}$ and $A^{\text {assoc }}$ are the free energy contributions due to the ideal, 
monomer, and association interactions, respectively. The theory has been presented in previous work [49] and so only an overview of the approach is described below.

The contribution to the Helmholtz free energy due to the monomer fluid is given as a combination of the dispersion and electrostatic interactions between the segments as:

$$
\frac{A^{\text {mono }}}{N k_{b} T}=\frac{A^{s w}}{N k_{b} T}+\frac{A^{e l}}{N k_{b} T}
$$

where, $A^{\text {sw }}$ and $A^{e l}$ are the free energy contributions due to square-well dispersion interaction and electrostatic interactions, respectively.

$$
\frac{A^{s w}}{N k_{b} T}=\left(\sum_{i=1}^{n} x_{i} m_{i}\right) \frac{A^{s w}}{N_{s} k_{b} T}=\left(\sum_{i=1}^{n} x_{i} m_{i}\right) a^{s w}
$$

$a^{s w}$ is the contribution to free energy due to the attractive square-well dispersive interactions between monomer segments and is obtained as in the original SAFT-VR approach from a second order high temperature perturbation expansion [34]. The contribution to the Helmholtz free energy from the electrostatic interactions, $A^{e l}$, is obtained from Blum and Wei's [41] solution to the Ornstein-Zernike equation using integral theory approach within the NPMSA closure for a mixture of ions and dipole of arbitrary size, and is given by,

$$
\frac{A^{e l}}{V k_{b} T}=\frac{E^{e l}}{V k_{b} T}-J-J^{\prime}
$$

where, $V$ is the total volume of the solution, $J$ and $J^{\prime}$ are the virial integral terms and $E^{e l} / V k_{b} T$ is the internal energy per unit volume defined as,

$$
\frac{E^{e l}}{V k_{b} T}=\frac{1}{4 \pi}\left\{\alpha_{0}^{2} \sum_{k=1}^{2} \rho_{k} z_{k} N_{k}-2 \alpha_{2} \alpha_{0} \rho_{n} B^{10}-\frac{2 \alpha_{2}^{2} \rho_{n} b_{2}}{\sigma_{n}^{3}}\right\}
$$


with virial integral terms $J$ and $J^{\prime}$ defined as [50],

$$
\begin{gathered}
J=\frac{1}{12 \pi}\left\{\alpha_{0}^{2} \sum_{k=1}^{2} \rho_{k} z_{k} N_{k}-2 \alpha_{0} \alpha_{2} \rho_{n} B^{10}-\frac{6 \alpha_{2}^{2} \rho_{n} b_{2}}{\sigma_{n}^{3}}\right\} \\
J^{\prime}=\frac{\pi}{3} \sum_{i} \sum_{j} \rho_{i} \rho_{j} \sigma_{i j}\left\{\sum_{m n l} \frac{(-1)^{l}}{2 l+1}\left[g_{i j}^{m n l}\left(\sigma_{i j}\right)\right]^{2}-\left[g_{i j}^{h s}\left(\sigma_{i j}\right)\right]^{2}\right\}
\end{gathered}
$$

where $\alpha_{0}$ and $\alpha_{2}$ are the ion-ion coupling and dipole-dipole interaction energy strength parameters, $g_{i j}^{n m l}\left(\sigma_{i j}\right)$ is the radial distribution function (RDF) of ion/ dipolar species at contact and $g_{i j}^{h s}\left(\sigma_{i j}\right)$ is the hard-sphere RDF at contact. The parameters $B^{10}$ and $b_{2}$ corresponds to the ion-dipole and dipole-dipole interactions respectively, and are obtained from the solution of Ornstein-Zernike equation given by Wei and Blum [41]. The detailed description of each term in the expression of $A^{e l}$ is reported in previous work $[41,42]$.

The Helmholtz free energy contribution due to the formation of a chain containing homogeneous monomeric segments is given by,

$$
\frac{A^{\text {chain }}}{N k_{b} T}=\sum_{i=1}^{n_{\text {solvent }}} x_{i}\left(1-m_{i}\right) \ln g_{i i}^{M}\left(\sigma_{i i}\right)
$$

where, the sum is over all solvent compounds in the mixture and $g^{M}$ represents the contact value of the RDF of monomer segments. Since solvent molecules are described as dipolar SW molecules, the required $g^{M}$ is that of the dipolar square well fluid, i.e., $g^{D S W}$ and is obtained from the linearized version of the exponential (LEXP) approximation [51]. As shown in previous work [51], the LEXP approximation for the RDF is more 
accurate than the MSA, as it provides a better description of PVT and VLE properties of dipolar dispersive square well chain fluids.

The contribution to the free energy due to short-ranged attractive association interactions between solvent molecules is given by the usual SAFT expressions [52].

\section{Simulation}

Monte Carlo simulations in the isothermal-isobaric (NPT) ensemble have been performed to study the PVT behavior of several model mixed dipolar solvent electrolyte systems. Each simulation was started from an initial configuration in which 256 molecules were placed in random orientations on the sites of a face centered cubic lattice in a simulation box with periodic boundary conditions. The long-range charge-charge, charge-dipole, and dipole-dipole interactions between polar solvents and charged ions were captured through the reaction field method [53-56]. The reaction field method has shown to be as accurate as the Ewald sum for calculating long-range dipolar and electrostatic forces [57-60]. In the reaction field method, the long-range interactions are truncated at a finite cut-off distance from each ion and dipolar molecule, and replaced by a dielectric continuum. The effect of the dielectric continuum is taken into account by including an additional term into the long-range charge-charge, charge-dipole, and dipole-dipole interaction potentials. In this work, the cut-off distance $\left(r_{c}\right)$ has been set to $3 \sigma$, beyond which the pair potential is zero and a dielectric continuum $\varepsilon_{R F}$ applied.

In the systems included in this study, the solvent molecules are highly associative. This kind of strong association interaction between molecules can promote the formation of stable clusters, making the complete sampling of phase space challenging [61-63]. To 
avoid poor sampling, several biasing schemes have been proposed in the literature $[61$, 62, 64-66]. For example, the association-biased Monte Carlo (ABMC) [67] method, which strategically biases the configuration site where association is likely to occur, was the first biasing scheme to be proposed however it requires the determination of bonding regions, making the algorithm rather complex. Subsequently, Tsangaris et al. developed the bond bias Monte Carlo method for the sampling of dimer clusters [61] and Visco et al. [62] proposed the monomer-addition-subtraction algorithm for the study of linear and ring aggregates. More recently, Chen et al. [63] proposed the aggregation volume bias Monte Carlo (AVBMC) biasing scheme, which can be applied to any cluster architecture (i.e., it is not restricted to the sampling of dimers, chains or rings) and is computationally more efficient as the identification of clusters is not required. In the simulations reported here, although systems with low association energy (i.e., $\varepsilon^{H B}<10 \varepsilon$ ) are studied and so are outside of range in which regular GEMC simulations are expected to fail [61], we have used the AVBMC biasing scheme developed by Chen et. al. [63] to avoid potential issues of poor sampling and increase the reliability of the PVT data

In the simulations the potential model for the association interactions is given by,

$$
\begin{aligned}
& u\left(r_{12} ; \theta_{i}, \theta_{j}\right) \\
& =\left\{\begin{array}{cc}
\varepsilon^{H B}, & \sigma \leq r_{i j}<r_{12} \text { and }\left|\theta_{i}\right|<\theta_{c} \text { and }|\pi-| \theta_{j}||<\theta_{c} \\
0 & \text { otherwise, }
\end{array}\right.
\end{aligned}
$$

where, $\theta_{i}$ and $\theta_{j}$ are the angles between the direction vectors and the center-to-center vector of atoms $i$ and $j$ respectively. Different association sites residing upon the same or different molecules can interact with a short-ranged associating energy of magnitude $\varepsilon^{H B}$. Although, in Wertheim's theory, bonding is limited at each association site to dimers, 
higher order cluster formation is possible depending upon the size of the association site, strength of the site-site interactions, and the state conditions as illustrated by Docherty and Galindo [68]. In this work, the angular cut-off $\theta_{c}$ is set to $27^{\circ}$ in order to restrict bonding to dimer formation [22].

The AVBMC move has been used in conjunction with traditional Monte Carlo displacement, rotation and volume moves. In a single simulation cycle, $\mathrm{N}$ trial displacement, rotations, and AVBMC moves along with one volume change move has been employed. The extent of displacement, reorientation, AVBMC and volume trial moves has been adjusted so that individual acceptance probabilities are between $20-30 \%$. An initial simulation of 500000-1000000 cycles was performed to equilibrate the system before averaging for between 1000000 and 2000000 cycles. In order to examine whether equilibrium had been reached or not, several simulations from different initial configurations were performed and results for the thermodynamic properties compared. For each system studied, the packing fraction and system energy at a given reduced pressure are reported and were obtained as ensemble averages and the errors estimated by taking the standard deviation, as given by following equation,

$$
\text { Error }=\sqrt{\frac{1}{M} \sum_{n=1}^{M} I^{* 2}-\left\langle\frac{1}{M} \sum_{n=1}^{M} I^{*}\right\rangle^{2}}
$$

where, $I$ is calculated packing fraction/ energy and $M$ number of cycles.

\section{Results and discussion}


A complete list of systems studied by Monte Carlo simulation is provided in Table 1 . In systems 1 and 2, the effect of solvent polarity on the PVT behavior is evaluated while systems 1, 3 and 4 enable the effect of different ionic concentrations on the PVT behavior to be examined. In order to take that into account the effect of solvent composition, in systems 1,5 and 6 we consider different ratios of monomer and dimer solvent molecules. We note that systems 1-6 are symmetric in nature as all the ions and molecules have the same segment diameter; however, in experimental mixed solvent electrolyte systems the ions and solvent molecules are of different sizes. Therefore the effect of ion and solvent molecule size on the thermodynamics of the fluid has been tested in systems 7 and 8 . The results of the NPT Monte Carlo simulations are provided in Table 2.

TABLE I. Model parameters for the electrolyte fluids studied. $\sigma_{d 1}, \sigma_{d 2}, \sigma^{*+}$ and $\sigma^{*_{-}}$are the reduced diameter of the solvent 1 (monomer), solvent 2 (dimer), cation and anion molecules respectively. $\mu_{d 1}^{* 2}$ and $\mu_{d 2}^{* 2}$ are the reduced squared dipole moments of solvent 1 and solvent 2 , where $\mu^{* 2}=\mu^{2} / k_{b} T \sigma^{3}, \varepsilon^{*}$ is the reduced depth of the square-well potential, $\lambda$ the range of the potential, $\psi^{*}$ the reduced association energy, $r_{c}^{*}$ the reduced association cutoff radius, $N_{\text {ion }}$ the number of ions, and $N_{\text {solvent } 1}, N_{\text {solvent } 2}$ the number of monomer and dimer solvent molecules.

\begin{tabular}{|c|c|c|c|c|c|c|c|c|c|c|c|c|c|}
\hline System & $\sigma_{d 1}^{*}$ & $\sigma_{d 2}^{*}$ & $\sigma_{+}^{*}$ & $\sigma_{-}^{*}$ & $\mu_{d 1}^{* 2}$ & $\mu_{d 2}^{* 2}$ & $\varepsilon^{*}$ & $\lambda$ & $\psi^{*}$ & $r_{c}^{*}$ & $N_{\text {ion }}$ & $N_{\text {solvent1 }}$ & $N_{\text {solvent } 2}$ \\
\hline 1 & 1 & 1 & 1 & 1 & 1 & 2 & 1 & 1.5 & 5 & 1.05 & 8 & 124 & 124 \\
\hline 2 & 1 & 1 & 1 & 1 & 1 & 4 & 1 & 1.5 & 5 & 1.05 & 8 & 124 & 124 \\
\hline 3 & 1 & 1 & 1 & 1 & 1 & 2 & 1 & 1.5 & 5 & 1.05 & 4 & 126 & 126 \\
\hline 4 & 1 & 1 & 1 & 1 & 1 & 2 & 1 & 1.5 & 5 & 1.05 & 16 & 120 & 120 \\
\hline 5 & 1 & 1 & 1 & 1 & 1 & 2 & 1 & 1.5 & 5 & 1.05 & 8 & 200 & 48 \\
\hline 6 & 1 & 1 & 1 & 1 & 1 & 2 & 1 & 1.5 & 5 & 1.05 & 8 & 4 & 244 \\
\hline 7 & 1 & $4 / 3$ & 1 & 1 & 1 & 2 & 1 & 1.5 & 5 & 1.05 & 8 & 124 & 124 \\
\hline 8 & 1 & $4 / 3$ & $2 / 3$ & $5 / 3$ & 1 & 2 & 1 & 1.5 & 5 & 1.05 & 8 & 124 & 124 \\
\hline
\end{tabular}

In Figure 2, a comparison has been made between SAFT-VR+DE theoretical predictions and Monte Carlo NPT ensemble simulation results for the PVT behavior of systems 1 and 2 . From the figure we can see that over the range of temperatures studied $\left(T^{*}=1.2,1.4,1.6,1.8\right)$, the theory is found to be in good agreement with the simulation 
results. In system 2, compared to system 1, the polarity of dimer molecules increases (from $\mu_{d 2}^{* 2}=2$ to 4 ), which results in increased attractive interactions between the solvent molecules and an increase in the density at a fixed pressure. Although the theory correctly captures this trend, we note that at higher temperatures for system 2 the theory under predicts the density as shown in figure $2 \mathrm{~b}$; however, the deviations are well within the errors as reported in table 2.
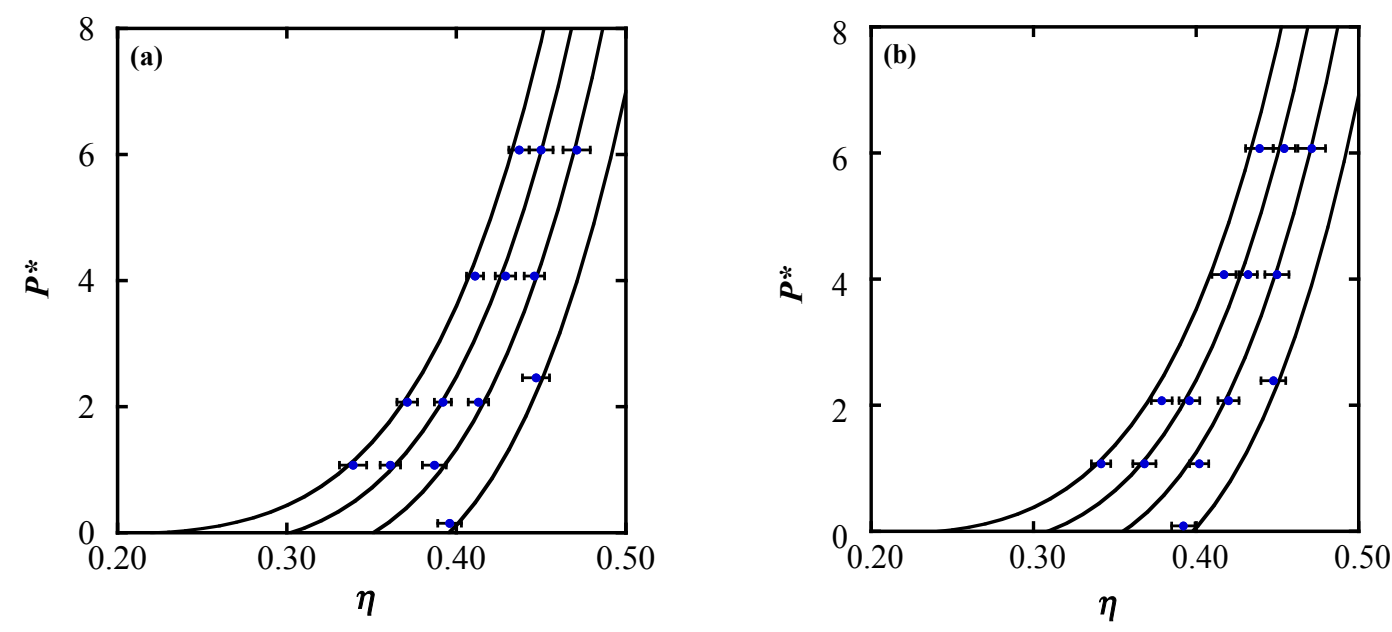

Figure 2. Comparison of predictions from the SAFT-VR + DE equation and NPT Monte Carlo simulation results for symmetric electrolyte solutions with $\varepsilon^{*}=1.0, \lambda=1.5, \sigma^{+^{*}}=\sigma^{-*}=\sigma_{d 1}^{*}=\sigma_{d 2}^{*}=1.0, \psi^{*}=5.0$, $r_{c}^{*}=1.05$, charge $q=1$, ion concentration of $8 / 256$, and (a) dipole moment $\mu_{d 1}^{* 2}=0.5$ and $\mu_{d 2}^{* 2}=2.0$ at $\mathrm{T}^{*}=k_{b} T / \varepsilon=1.2,1.4,1.6$ and 1.8 for system 1 and (b) dipole moment $\mu_{d 1}^{* 2}=0.5$ and $\mu_{d 2}^{*_{2}}=4.0$ at $\mathrm{T}^{*}=k_{b} T / \varepsilon=1.2,1.4,1.6$, and 1.8 for system 2 . The solid lines represent predictions from the SAFT-VR + DE equation and the squares represent the NPT Monte Carlo simulation data. 

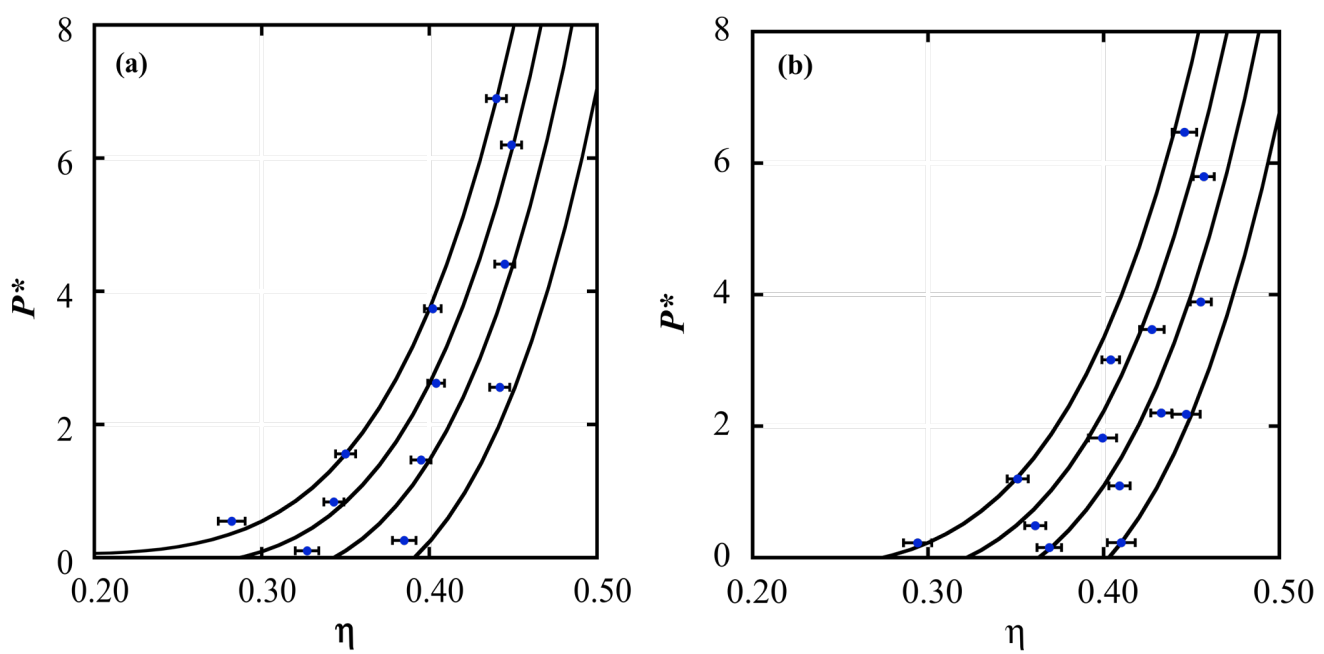

Figure 3. Comparison of predictions from the SAFT-VR + DE equation and NPT Monte Carlo simulation results for symmetric electrolyte solutions with $\varepsilon^{*}=1.0, \lambda=1.5, \sigma^{+^{*}}=\sigma^{-*}=\sigma_{d 1}^{*}=\sigma_{d 2}^{*}=1.0, \psi^{*}=5.0$, $r_{c}^{*}=1.05$, charge $q=1$, dipole moment $\mu_{d 1}^{* 2}=0.5$ and $\mu_{d 2}^{* 2}=2.0$ at $\mathrm{T}^{*}=k_{b} T / \varepsilon=1.2,1.4,1.6$ and 1.8 for ion concentration of (a) $4 / 256$, and (b) $16 / 256$, with $N_{\text {solvent } 1}=N_{\text {solvent } 2}$. The solid lines represent predictions from the SAFT-VR + DE equation and the squares represent the NPT Monte Carlo simulation data.

Systems 1 and 2 contain 8 ions ( 4 cations and 4 anions) along with 248 solvent (both monomer and dimer) molecules. In experimental electrolyte systems, thermodynamic properties vary as a function of ion concentration. In order to test whether the proposed approach can capture the effect of changes in ion concentration systems 3 and 4 are studied in which the ion concentrations are $0.79 \%(4 / 256)$ and $3.2 \%$ (16/256) mol \% of salt respectively. We note for comparison that $0.79 \%$ and $3.2 \%$ corresponds to 0.344 and $1.446 \mathrm{~m}$, respectively for a methanol + water $+\mathrm{NaCl}$ solution. The highest tested molality of $1.446 \mathrm{~m}$ is comparable to experimental mixed solvent electrolyte systems as the majority of the available experimental data is below $1.5 \mathrm{~m}$. The 
results for systems 3 and 4 are presented in figure 3, from which we can see that the theoretical predictions and Monte Carlo simulation results are in good agreement for both systems. As the concentration of the ions increases, the density at a given pressure and temperature increases, due to the increased attractive interaction. However, at higher ionic concentrations, the SAFT-VR+DE equation slightly over predicts the pressure, perhaps due to approximating the ion-dipolar interactions through those of an effective fluid.

In addition to the concentration of ions in electrolyte solutions, the composition of the solvent in mixed solvent electrolyte systems can play an important role in determining the thermodynamic properties [69]. For example, in the measurement of mean ionic activity coefficients for $\mathrm{NaBr}+$ ethanol + water the ionic activity was found to change from 0.6643 to 0.2951 at 0.31 salt molality $(m)$ with a change in the solvent ratio between water and ethanol from 9:1 to 1:9 [69]. To examine this effect. in systems 5 and 6 the ratio of the dimer and monomer solvent is varied from the 50:50 mixture used in systems $1-4$ (i.e., in systems $1-4$, the solvent molecules are divided equally between the monomer and dimers molecule). In system 5 200/248 and in system 6 4/248 particles are considered monomers, whereas the number of ions is maintained at 8 in both cases. The polarity of the systems studied also increases as the number of dimers in the system increases, due to the higher dipole moment in the dimer molecules compared to the monomer molecules. As a result, as shown in Figure 4, the density of system 6 is higher at a given temperature and pressure than for system 5. For system 1 with a 50:50 mixture of dimer and monomer solvent species the solution density lies as expected in between system 5 and 6 at a specific temperature and pressure. From figure 4, it can also be seen 
that the theory is able to capture this change in the PVT behavior and is in good agreement with the simulation results.
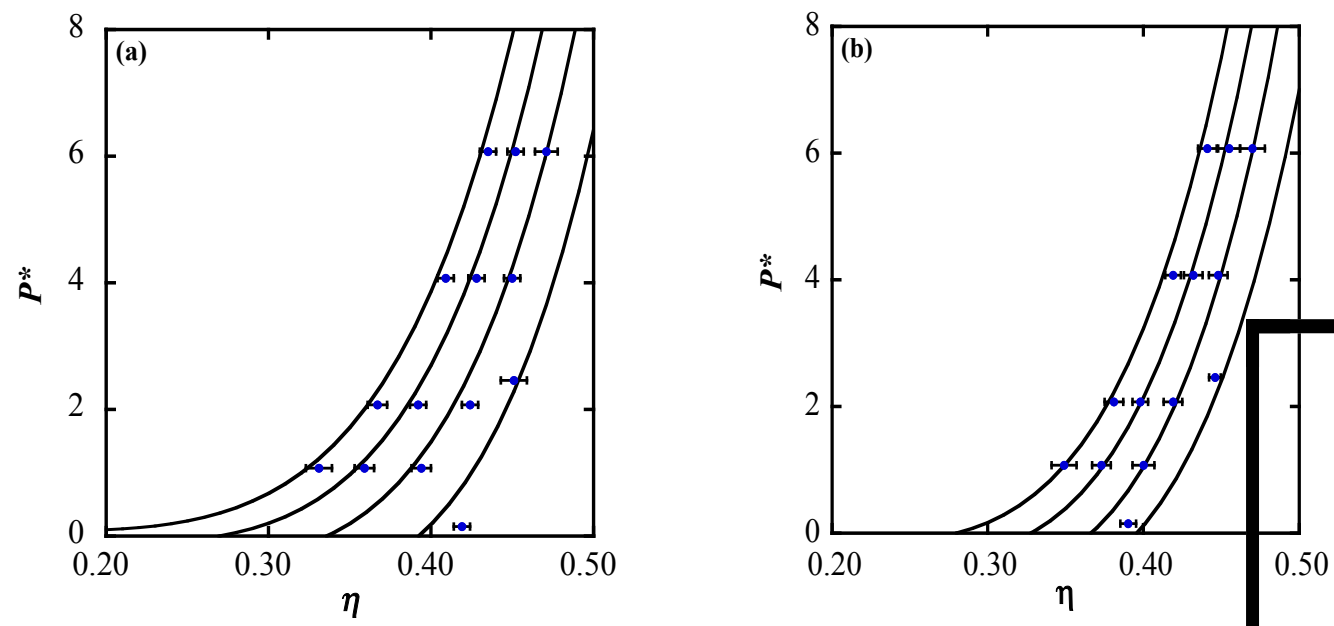

Figure 4 Comparison of predictions from the SAFT-VR + DE equation and NPT Monte Carlo simulation results for symmetric electrolyte solutions with $\varepsilon^{*}=1.0, \lambda=1.5, \sigma^{+^{*}}=\sigma^{-*}=\sigma_{d 1}^{*}=\sigma_{d 2}^{*}=1.0, \psi^{*}=5.0$, $r_{c}^{*}=1.05$, charge $q=1$, dipole moment $\mu_{d 1}^{* 2}=0.5$ and $\mu_{d 2}^{* 2}=2.0$, ion concentration of $8 / 256$ at $\mathrm{T}^{*}=k_{b} T / \varepsilon=1.2,1.4,1.6$ and 1.8 for different solvent rations. (a) 200 particles of monomers and 48 of dimers; (b) 4 particles of monomers and 244 of dimers. The solid lines represent predictions from the SAFT-VR + DE equation and the squares represent the NPT Monte Carlo simulation data.

To more closely mimic experimental systems, we now consider systems (7 and 8) in which the ions and dipolar solvent molecules have different diameters. Experimentally, electrolyte solutions often contain highly asymmetric species, for example the $\mathrm{LiBr}+$ ethanol + water mixture in which $\mathrm{Li}^{+}$has an ionic diameter of 1.2 compared to $\mathrm{Br}^{-}$having an ionic diameter of 3.92 and solvents water and ethanol are of different sizes and polarities. It is therefore desirable to be able to capture the effect of asymmetry on the PVT behavior. In system 7, while the ions have the same diameter, the solvent molecules 
are asymmetric in nature, with a ratio of $3: 4$ between the monomer and dimer molecules. System 8 is composed of completely asymmetric species, with the ratio of monomer, dimer, cation and anion diameters being 3:4:2:5. The results for both systems are presented in figure 5, where again we see that the SAFT-VR+DE approach is in good agreement with the simulation data. Comparison between systems 1, 7, and 8 (Figs. 2a, 5a, and 5b) show that the asymmetric system (system 8) exhibits the highest density at a given pressure and temperature, while the symmetric system (system1) has the lowest density.
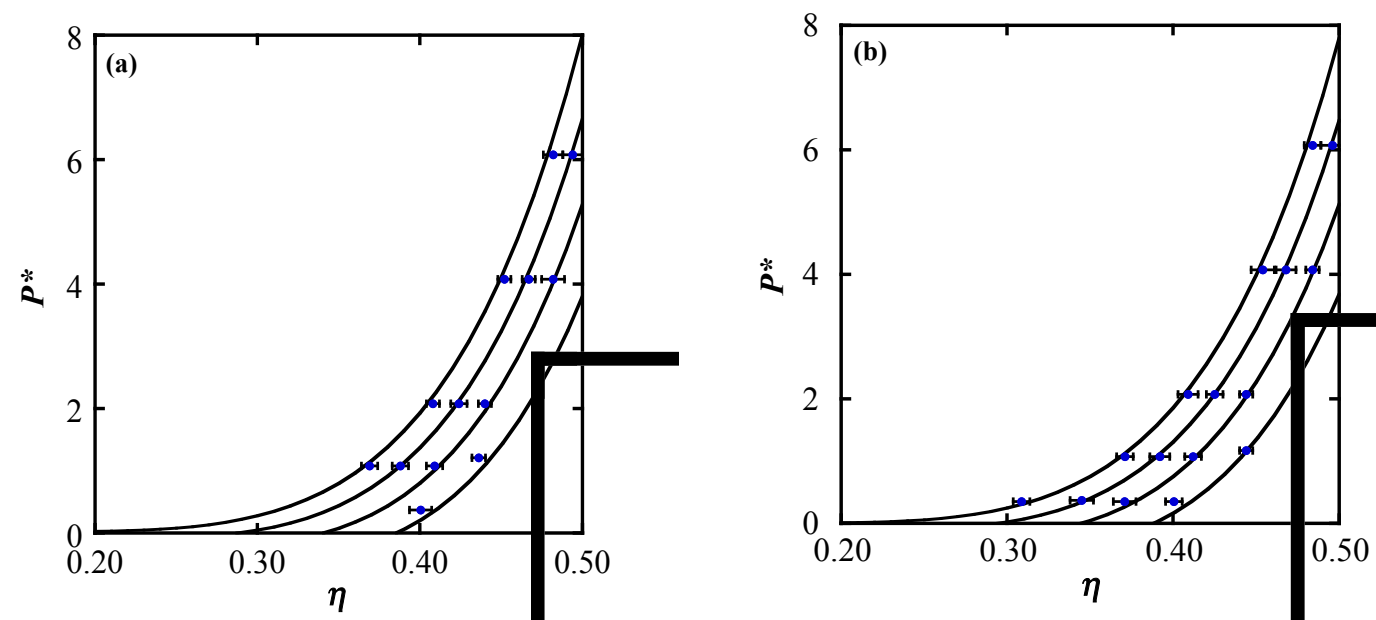

Figure 5. Comparison of predictions from the SAFT-VR + DE equation and NPT Monte Carlo simulation results for asymmetric electrolyte solutions with $\varepsilon^{*}=1.0, \lambda=1.5, \psi^{*}=5.0, r_{c}^{*}=1.05$, charge $q=1$, dipole moment $\mu_{d 1}^{*_{2}}=0.5$ and $\mu_{d 2}^{* 2}=2.0$, ion concentration of $8 / 256$ at $\mathrm{T}^{*}=k_{b} T / \varepsilon=1.2,1.4,1.6$ and 1.8 for different solvent and ion sizes. (a) $\sigma^{+^{*}}=\sigma^{-^{*}}=\sigma_{d 1}^{*}=1.0, \sigma_{d 2}^{*}=1.333$; (b) $\sigma^{+^{*}}=0.667 \sigma^{-*}=1.667$ $\sigma_{d 1}^{*}=1.0 \sigma_{d 2}^{*}=1.333$. The solid lines represent predictions from the SAFT-VR + DE equation and the squares represent the NPT Monte Carlo simulation data. 


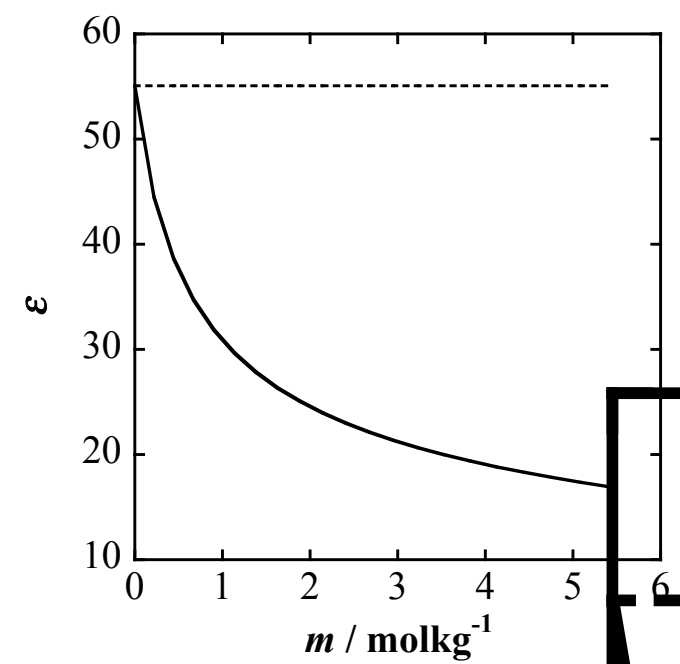

Figure 6 Comparison of the ion concentration and solvent composition dependent dielectric constant (solid line) with the solvent concentration dependent dielectric constant calculated predicted from the SAFT$\mathrm{VR}+\mathrm{D}$ equation (dotted line) for $\mathrm{NaBr}+$ water $+(50$ weight $\%)$ methanol mixed solvent electrolyte systems at $298.15 \mathrm{~K}$ and 1.01325 bar. The system parameters are given in table 3.

Having seen that the non primitive model based SAFT-VR+DE equation of state is able to accurately predict the effect of long-range electrostatic interactions on the thermodynamics of mixed dipolar solvent electrolyte solutions for both symmetric (seminon primitive model) and asymmetric (unrestricted-non primitive model) model systems, a comparison is now made with the primitive model of electrolytes to highlight the improved accuracy obtained through an implicit representation of solvent species as in the SAFT-VR+DE equation. Since the primitive model employs an implicit solvent the dielectric constant is an input to the calculations and therefore dependent on available experimental data. Although ion concentration in the solution is known to have a significant influence on the solution dielectric [70], with the dielectric constant decreasing as the salt concentration increases, experimental dielectric data as a function of ion concentration is not available for mixed solvent electrolyte systems and so 
primitive model approaches must use solvent composition based experimental dielectric constant data that is independent of salt concentration. In the figure 6 , the SAFT-VR+DE prediction for the influence of the ion concentration on the dielectric constant for the $\mathrm{NaBr}+$ methanol + water system is presented. As can be seen from the figure, the theory predicts a significant reduction in the dielectric constant with increasing salt molality at 50 weight $\%$ composition of methanol-water. Although no experimental data is available for verification, the trend predicted by the SAFT-VR+DE equation is consistent with that observed in experimental studies of aqueous (i.e., single solvent) electrolyte systems [70].

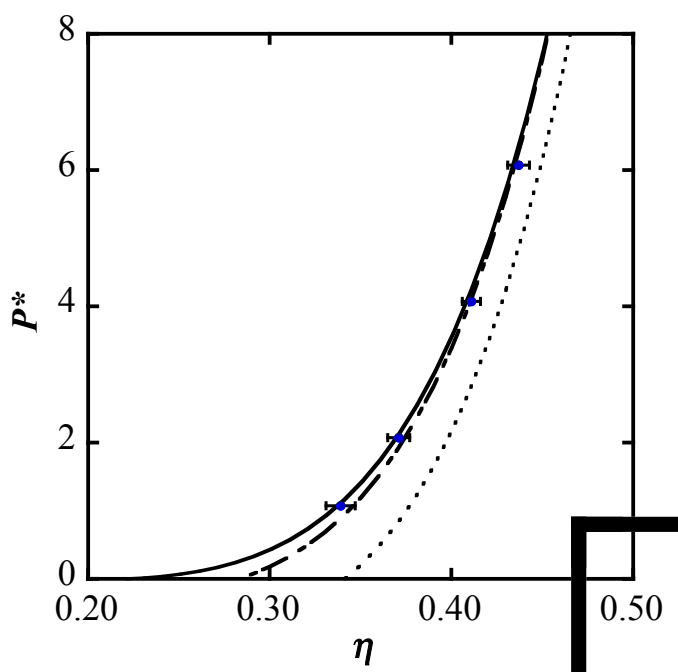

Figure 7. Comparison of predictions from the SAFT-VR + DE equation and NPT Monte Carlo simulation data for symmetric electrolyte solutions with $\varepsilon^{*}=1.0, \lambda=1.5, \sigma^{+^{*}}=\sigma^{-*}=\sigma_{d 1}^{*}=\sigma_{d 2}^{*}=1.0, \psi^{*}=5.0, r_{c}^{*}$ $=1.05$, charge $q=1$, dipole moment $\mu_{d 1}^{*_{2}}=0.5$ and $\mu_{d 2}^{*_{2}}=2.0$, and ion concentrations of $8 / 256(124$ monomers and 124 dimers) at $T^{*}=k_{b} T / \varepsilon=1.8$. The solid line represents predictions from the nonprimitive model based SAFT-VR + DE, dotted line represents predictions from the primitive model + SAFT-VR using a solvent composition based (and salt concentration independent) dielectric constant, dashdotted line represents predictions form the primitive model + SAFT-VR using a salt concentration and solvent composition dependent dielectric constant, and the squares are NPT Monte Carlo simulation results. 


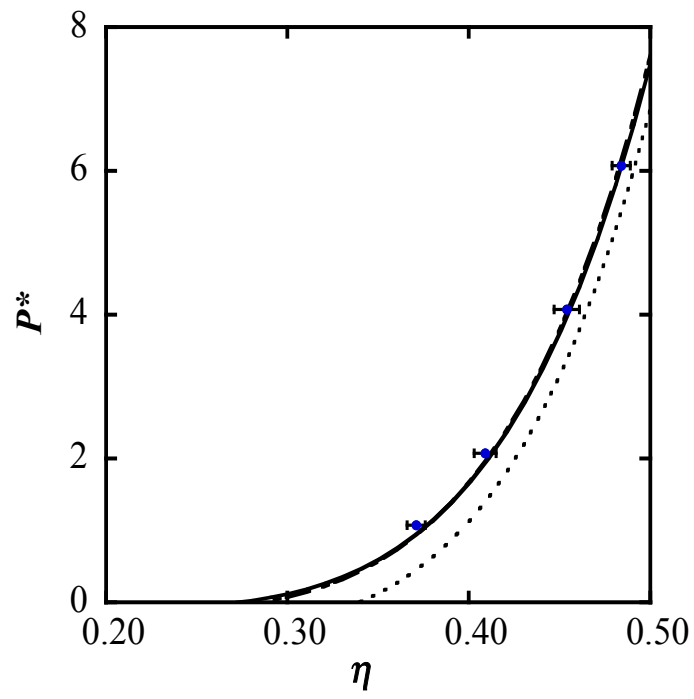

Figure 8. Comparison of predictions from the SAFT-VR + DE equation and NPT Monte Carlo simulation data for asymmetric electrolyte solutions with $\varepsilon^{*}=1.0, \lambda=1.5, \sigma^{+^{*}}=0.667, \sigma^{-*}=1.667, \sigma_{d 1}^{*}=1.0, \sigma_{d 2}^{*}$ $=1.333, \psi^{*}=5.0, r_{c}^{*}=1.05$, charge $q=1$, dipole moment $\mu_{d 1}^{* 2}=0.5$ and $\mu_{d 2}^{* 2}=2.0$, and ion concentrations of 8 / 256 (124 monomers and 124 dimers) at $T^{*}=k_{b} T / \varepsilon=1.8$. The solid line represents predictions from the non-primitive model based SAFT-VR + DE, dotted line represents predictions from the primitive model + SAFT-VR using a solvent composition based (and salt concentration independent) dielectric constant, dash-dotted line represents predictions form the primitive model + SAFT-VR using a salt concentration and solvent composition dependent dielectric constant, and the squares are NPT Monte Carlo simulation results.

To quantify the effect of an ionic concentration based dielectric constant on the predictive ability of an equation of state a comparison has been made between the nonprimitive and primitive models, both with and with out the salt concentration dependent dielectric constant in figure 7 for system 1 at $\mathrm{T}^{*}=1.8$. From the figure we can see that the primitive model with a salt concentration independent dielectric constant shows 
significant deviations from the simulated PVT results, whereas the non-primitive model provides an accurate prediction. We note that, when the input dielectric constant of the primitive model is changed to a salt concentration dependent one (obtained as an output from the non-primitive model), the primitive model shows immediate improvement in predictive ability and the result is comparable in accuracy with the non-primitive model. Similar trends are observed for the asymmetric system (system 8) as presented in the figure 8 , i.e., the theoretical predictions of the PVT behavior from the primitive model significantly improve with the use of a salt concentration dependent dielectric constant, with the resulting accuracy being comparable to that of the non-primitive model. The use of the non-primitive model in the development of equations of state for mixed solvent electrolytes is therefore advantageous as it requires no prior knowledge of the appropriate dielectric constant in order to capture changes in the dielectric constant with salt concentration; such information can be hard to obtain for mixed solvent electrolyte systems.

TABLE 2. NPT Monte Carlo simulation results along with errors for systems $1-8$. The reduced temperature is given by $T^{*}=k_{B} T / \varepsilon$, reduced pressure by $P^{*}=P \sigma^{3} / \varepsilon$ and reduced energy by

$$
E^{*}=E / N \varepsilon \text {. }
$$

\begin{tabular}{|c|c|c|c|c|c|c|}
\hline System & $T^{*}$ & $P^{*}$ & $\eta$ & Error & $E^{*}$ & Error \\
\hline \multirow[t]{14}{*}{1} & \multirow[t]{2}{*}{1.2} & 0.15 & 0.396 & 0.007 & -12.029 & 0.513 \\
\hline & & 2.46 & 0.447 & 0.008 & -12.841 & 0.537 \\
\hline & \multirow[t]{4}{*}{1.4} & 1.0733 & 0.387 & 0.007 & -11.711 & 0.387 \\
\hline & & 2.0733 & 0.413 & 0.006 & -12.008 & 0.373 \\
\hline & & 4.0733 & 0.446 & 0.006 & -12.282 & 0.313 \\
\hline & & 6.0733 & 0.471 & 0.008 & -12.655 & 0.393 \\
\hline & \multirow[t]{4}{*}{1.6} & 1.0733 & 0.361 & 0.006 & -11.003 & 0.502 \\
\hline & & 2.0733 & 0.392 & 0.005 & -11.618 & 0.409 \\
\hline & & 4.0733 & 0.429 & 0.006 & -11.931 & 0.446 \\
\hline & & 6.0733 & 0.450 & 0.007 & -12.662 & 0.317 \\
\hline & \multirow[t]{4}{*}{1.8} & 1.0733 & 0.339 & 0.008 & -9.926 & 0.294 \\
\hline & & 2.0733 & 0.371 & 0.006 & -11.118 & 0.520 \\
\hline & & 4.0733 & 0.411 & 0.005 & -11.738 & 0.345 \\
\hline & & 6.0733 & 0.437 & 0.006 & -11.950 & 0.305 \\
\hline 2 & 1.2 & 0.086 & 0.392 & 0.007 & -12.588 & 0.609 \\
\hline
\end{tabular}




\begin{tabular}{|c|c|c|c|c|c|c|}
\hline & & 2.3900 & 0.448 & 0.008 & -13.860 & 0.559 \\
\hline & \multirow{4}{*}{1.4} & 1.0733 & 0.402 & 0.006 & -12.593 & 0.368 \\
\hline & & 2.0733 & 0.420 & 0.006 & -12.725 & 0.387 \\
\hline & & 4.0733 & 0.450 & 0.007 & -13.051 & 0.367 \\
\hline & & 6.0733 & 0.471 & 0.009 & -14.002 & 0.601 \\
\hline & \multirow{4}{*}{1.6} & 1.0733 & 0.368 & 0.007 & -11.472 & 0.557 \\
\hline & & 2.0733 & 0.396 & 0.006 & -12.301 & 0.559 \\
\hline & & 4.0733 & 0.432 & 0.006 & -13.205 & 0.427 \\
\hline & & 6.0733 & 0.454 & 0.007 & -13.279 & 0.481 \\
\hline & \multirow{4}{*}{1.8} & 1.0733 & 0.341 & 0.007 & -10.860 & 0.359 \\
\hline & & 2.0733 & 0.379 & 0.006 & -11.560 & 0.292 \\
\hline & & 4.0733 & 0.417 & 0.005 & -12.461 & 0.389 \\
\hline & & 6.0733 & 0.439 & 0.006 & -12.665 & 0.279 \\
\hline \multirow{12}{*}{3} & \multirow{2}{*}{1.2} & 0.260 & 0.385 & 0.007 & -8.537 & 0.242 \\
\hline & & 2.560 & 0.442 & 0.006 & -9.516 & 0.259 \\
\hline & \multirow{3}{*}{1.4} & 0.105 & 0.327 & 0.009 & -7.178 & 0.245 \\
\hline & & 1.467 & 0.395 & 0.005 & -8.139 & 0.318 \\
\hline & & 4.410 & 0.445 & 0.006 & -9.094 & 0.302 \\
\hline & \multirow{3}{*}{1.6} & 0.838 & 0.343 & 0.007 & -7.168 & 0.237 \\
\hline & & 2.619 & 0.404 & 0.005 & -8.247 & 0.244 \\
\hline & & 6.200 & 0.449 & 0.006 & -8.939 & 0.273 \\
\hline & \multirow{4}{*}{1.8} & 0.549 & 0.282 & 0.008 & -6.154 & 0.192 \\
\hline & & 1.558 & 0.350 & 0.006 & -7.185 & 0.196 \\
\hline & & 3.740 & 0.402 & 0.005 & -8.038 & 0.201 \\
\hline & & 6.895 & 0.440 & 0.006 & -8.645 & 0.244 \\
\hline \multirow{13}{*}{4} & \multirow{2}{*}{1.2} & 0.23 & 0.410 & 0.008 & -19.677 & 0.961 \\
\hline & & 2.183 & 0.447 & 0.008 & -20.440 & 0.840 \\
\hline & \multirow{3}{*}{1.4} & 0.154 & 0.369 & 0.008 & -19.116 & 0.935 \\
\hline & & 1.094 & 0.413 & 0.007 & -20.307 & 0.900 \\
\hline & & 4.02 & 0.456 & 0.008 & -21.267 & 0.899 \\
\hline & \multirow{4}{*}{1.6} & 0.486 & 0.367 & 0.009 & -18.451 & 1.081 \\
\hline & & 1.82 & 0.401 & 0.006 & -19.821 & 0.844 \\
\hline & & 3.47 & 0.427 & 0.006 & -20.523 & 0.831 \\
\hline & & 5.794 & 0.458 & 0.008 & -21.725 & 1.076 \\
\hline & \multirow{4}{*}{1.8} & 0.228 & 0.303 & 0.007 & -17.269 & 1.017 \\
\hline & & 1.198 & 0.357 & 0.007 & -18.240 & 0.715 \\
\hline & & 3.35 & 0.419 & 0.006 & -20.066 & 0.721 \\
\hline & & 6.47 & 0.447 & 0.006 & -20.126 & 0.717 \\
\hline \multirow{14}{*}{5} & \multirow{2}{*}{1.2} & 0.15 & 0.419 & 0.005 & -16.477 & 0.264 \\
\hline & & 2.46 & 0.451 & 0.008 & -17.323 & 0.194 \\
\hline & \multirow{4}{*}{1.4} & 1.0733 & 0.394 & 0.006 & -14.590 & 0.194 \\
\hline & & 2.0733 & 0.424 & 0.005 & -15.218 & 0.174 \\
\hline & & 4.0733 & 0.450 & 0.005 & -15.798 & 0.183 \\
\hline & & 6.0733 & 0.471 & 0.007 & -16.117 & 0.198 \\
\hline & \multirow{4}{*}{1.6} & 1.0733 & 0.359 & 1.0733 & -13.074 & 0.175 \\
\hline & & 2.0733 & 0.392 & 2.0733 & -14.087 & 0.165 \\
\hline & & 4.0733 & 0.428 & 4.0733 & -15.034 & 0.255 \\
\hline & & 6.0733 & 0.452 & 6.0733 & -15.660 & 0.169 \\
\hline & \multirow{4}{*}{1.8} & 1.0733 & 0.331 & 0.008 & -12.871 & 0.191 \\
\hline & & 2.0733 & 0.367 & 0.006 & -13.626 & 0.169 \\
\hline & & 4.0733 & 0.409 & 0.005 & -14.105 & 0.161 \\
\hline & & 6.0733 & 0.435 & 0.005 & -14.977 & 0.157 \\
\hline 6 & 1.2 & 0.151 & 0.390 & 0.005 & -10.036 & 0.182 \\
\hline
\end{tabular}




\begin{tabular}{|c|c|c|c|c|c|c|}
\hline & & 2.46 & 0.446 & 0.003 & -10.861 & 0.344 \\
\hline & \multirow{4}{*}{1.4} & 1.0733 & 0.400 & 0.007 & -9.803 & 0.276 \\
\hline & & 2.0733 & 0.419 & 0.006 & -10.287 & 0.199 \\
\hline & & 4.0733 & 0.448 & 0.006 & -10.464 & 0.528 \\
\hline & & 6.0733 & 0.470 & 0.008 & -10.626 & 0.165 \\
\hline & \multirow{4}{*}{1.6} & 1.0733 & 0.373 & 0.006 & -9.081 & 0.188 \\
\hline & & 2.0733 & 0.398 & 0.005 & -9.778 & 0.255 \\
\hline & & 4.0733 & 0.432 & 0.006 & -10.104 & 0.889 \\
\hline & & 6.0733 & 0.455 & 0.007 & -10.246 & 0.192 \\
\hline & \multirow{4}{*}{1.8} & 1.0733 & 0.349 & 0.008 & -8.678 & 0.369 \\
\hline & & 2.0733 & 0.381 & 0.006 & -9.362 & 0.161 \\
\hline & & 4.0733 & 0.419 & 0.005 & -9.908 & 0.851 \\
\hline & & 6.0733 & 0.441 & 0.006 & -10.063 & 0.115 \\
\hline \multirow{13}{*}{7} & \multirow{2}{*}{1.2} & 0.37 & 0.404 & 0.005 & -11.371 & 0.183 \\
\hline & & 1.21 & 0.436 & 0.004 & -11.871 & 0.119 \\
\hline & \multirow{3}{*}{1.4} & 1.0773 & 0.409 & 0.005 & -10.930 & 0.190 \\
\hline & & 2.0773 & 0.441 & 0.004 & -11.532 & 0.109 \\
\hline & & 4.0773 & 0.482 & 0.011 & -11.870 & 0.728 \\
\hline & \multirow{4}{*}{1.6} & 1.0773 & 0.388 & 0.005 & -10.797 & 0.117 \\
\hline & & 2.0773 & 0.424 & 0.009 & -10.619 & 0.682 \\
\hline & & 4.0773 & 0.467 & 0.011 & -11.458 & 0.392 \\
\hline & & 6.0773 & 0.494 & 0.011 & -12.015 & 0.277 \\
\hline & \multirow{4}{*}{1.8} & 1.0773 & 0.369 & 0.009 & -9.679 & 0.159 \\
\hline & & 2.0773 & 0.408 & 0.008 & -10.392 & 0.347 \\
\hline & & 4.0773 & 0.452 & 0.009 & -10.869 & 0.689 \\
\hline & & 6.0773 & 0.482 & 0.011 & -10.963 & 0.531 \\
\hline \multirow{14}{*}{8} & \multirow{2}{*}{1.2} & 0.35 & 0.401 & 0.005 & -10.858 & 0.125 \\
\hline & & 1.17 & 0.435 & 0.004 & -11.096 & 0.172 \\
\hline & \multirow{4}{*}{1.4} & 0.35 & 0.371 & 0.006 & -10.217 & 0.189 \\
\hline & & 1.0733 & 0.412 & 0.005 & -10.638 & 0.118 \\
\hline & & 2.0733 & 0.443 & 0.004 & -11.060 & 0.146 \\
\hline & & 4.0733 & 0.483 & 0.003 & -12.357 & 0.298 \\
\hline & \multirow{4}{*}{1.6} & 0.35 & 0.345 & 0.007 & -9.653 & 0.162 \\
\hline & & 1.0733 & 0.392 & 0.005 & -10.338 & 0.161 \\
\hline & & 2.0733 & 0.425 & 0.008 & -10.644 & 0.468 \\
\hline & & 4.0733 & 0.468 & 0.009 & -11.188 & 0.501 \\
\hline & \multirow{4}{*}{1.8} & 0.35 & 0.309 & 0.010 & -8.799 & 0.480 \\
\hline & & 1.0733 & 0.371 & 0.008 & -9.318 & 0.469 \\
\hline & & 2.0733 & 0.409 & 0.008 & -10.13 & 0.537 \\
\hline & & 4.0733 & 0.454 & 0.008 & -10.85 & 0.485 \\
\hline
\end{tabular}


Table 3: SAFT-VR+DE parameters for methanol, $\mathrm{Na}$ and $\mathrm{Br}$ determined in this work. The reported parameters for water were taken from [43].

\begin{tabular}{|c|c|c|c|c|c|c|c|c|c|}
\hline & $\boldsymbol{\mu}(\mathbf{D})$ & $\boldsymbol{\sigma}(\AA)$ & $\boldsymbol{\varepsilon} / \mathbf{k}_{\mathbf{b}} \mathbf{( K )}$ & $\lambda$ & $\boldsymbol{m}$ & $\begin{array}{c}\boldsymbol{\varepsilon} \mathbf{\mathbf { H B }} / \mathbf{k}_{\mathbf{b}} \\
(\mathbf{K})\end{array}$ & $\begin{array}{c}\boldsymbol{K}^{\mathrm{HB}} \\
\left(\AA^{3}\right)\end{array}$ & $\varepsilon_{\text {ion- } \mathrm{H}_{2} \mathrm{O}} / k_{b}$ & $\varepsilon_{\text {ion-CH }} \mathrm{OH} / k_{b}$ \\
\hline $\mathbf{H}_{\mathbf{2}} \mathbf{O}$ & 2.179 & 3.003 & 312.36 & 1.52956 & 1 & 758.5521 & 1.5 & & \\
\hline $\mathbf{C H}_{3} \mathbf{O H}$ & 2.730 & 3.539 & 162.17 & 1.72229 & 1.2 & 1800.945 & 0.81 & & \\
\hline $\mathbf{N a}$ & & 2.8 & & & 1 & & & 1274.406 & 1915.47 \\
\hline $\mathbf{B r}$ & & 3.92 & & & 1 & & & & \\
\hline
\end{tabular}

\section{Conclusion}

In this work the SAFT-VR+DE approach, which was developed by combining the SAFTVR equation of state with the solution of the MSA within the non-primitive model, to provide an accurate representation of the free energy contribution due to electrostatic interactions in mixtures of ions and dipolar species, has been extended to the study of mixed dipolar solvent electrolytes. This is achieved using a one-fluid-like approximation in the ion-dipole and dipole-dipole terms. The approach has been extensively tested against PVT data obtained from NPT Monte Carlo simulations for a wide range of model mixed dipolar solvent electrolyte systems that vary in terms of the size of the ions and solvent molecules, polarity of solvent molecules, and salt and solvent composition. The theoretical approach is found to be in general in excellent agreement with the simulated PVT data illustrating that the effects of solvent and ionic size, polarity and composition on the thermodynamics of electrolyte solution are well captured by the theory. Comparisons were also made to predictions from the non primitive model to demonstrate that capturing the salt concentration dependence of the solvent dielectric is important in order to accurately predict the PVT behavior of mixed solvent electrolyte solutions. 


\section{Acknowledgements}

We gratefully acknowledge financial support from the National Science Foundation under Grant CBET-1067642 and the U.S. Department of Energy (DOE), Office of Basic Energy Sciences, Geoscience Research Program, through Grant No. ERKCC72 of Oak Ridge National Laboratory, which is managed for DOE by UT Battelle, LLC under Contract No. DE-AC05- 00OR22725.

\section{References}

[1] A. Anderko, P.M. Wang, M. Rafal, Electrolyte solutions: from thermodynamic and transport property models to the simulation of industrial processes, Fluid Phase Equilibr, 194 (2002) 123-142.

[2] C. Held, A. Prinz, V. Wallmeyer, G. Sadowski, Measuring and modeling alcohol/salt systems, Chemical Engineering Science, 68 (2012) 328-339.

[3] K.S. Pitzer, D.R. Schreiber, The Restricted Primitive Model for Ionic Fluids Properties of the Vapor and the Critical Region, Mol Phys, 60 (1987) 1067-1078.

[4] H.L. Friedman, Electrolyte solutions at equilibrium, Annual Review of Physical Chemistry, 32 (1981) 179-204.

[5] K.S. Pitzer, Thermodynamics of electrolytes. I. Theoretical basis and general equations, The Journal of Physical Chemistry, 77 (1973) 268--277.

[6] A.F. Bo Sander, Peter Rasmussen, Calculation of vapour-liquid equilibria in mixed solvent/salt systems using an extended UNIQUAC equation, Chemical Engineering Science, 41 (1986) 1171-1183.

[7] E.A. Macedo, Per Skovborg, and Peter Rasmussen, "Calculation of phase equilibria for solutions of strong electrolytes in solvent-water mixtures.", Chemical Engineering Science, 45 (1990) 875-882.

[8] I. Kikic, Maurizio Fermeglia, and Peter Rasmussen, Unifac prediction of vaporliquid equilibria in mixed solvent—-salt systems, Chemical engineering science 46 (1991) 2775-2780.

[9] J. Li, Hans-Martin Polka, and Jürgen Gmehling, A gE model for single and mixed solvent electrolyte systems: 1. Model and results for strong electrolytes, Fluid Phase Equilibr, 94 (1994) 89-114.

[10] H. Zerres, and J. M. Prausnitz, Thermodynamics of phase equilibria in aqueous organic systems with salt, Aiche J, 40 (1994) 676-691.

[11] Y. Liu, and Suphat Watanasiri, Representation of liquid-liquid equilibrium of mixed-solvent electrolyte systems using the extended electrolyte NRTL model, Fluid Phase Equilibr, 116 (1996) 193-200. 
[12] W.e.a. Yan, Prediction of vapor-liquid equilibria in mixed-solvent electrolyte systems using the group contribution concept, Fluid Phase Equilibr, 162 (1999) 97113.

[13] M.C. Iliuta, Kaj Thomsen, and Peter Rasmussen, Extended UNIQUAC model for correlation and prediction of vapour-liquid-solid equilibria in aqueous salt systems containing non-electrolytes. Part A. Methanol-water-salt systems, Chemical Engineering Science 55 (2000) 2673-2686.

[14] G.N.a.R. Lewis, Merle, The activity coefficient of strong electrolytes. 1, J Am Chem Soc, 43 (1921) 1112-1154.

[15] B. Sander, A. Fredenslund, P. Rasmussen, Calculation of Vapor-Liquid-Equilibria in Mixed-Solvent Salt Systems Using an Extended Uniquac Equation, Chemical Engineering Science, 41 (1986) 1171-1183.

[16] L.L. Lee, Thermodynamic consistency and reference scale conversion in multisolvent electrolyte solutions, J Mol Liq, 87 (2000) 129-147.

[17] M.J.E.D. Cardoso, J.P. Oconnell, Activity-Coefficients in Mixed-Solvent Electrolyte-Solutions, Fluid Phase Equilibr, 33 (1987) 315-326.

[18] W.D. Yan, M. Topphoff, C. Rose, J. Gemhling, Prediction of vapor-liquid equilibria in mixed-solvent electrolyte systems using the group contribution concept, Fluid Phase Equilibr, 162 (1999) 97-113.

[19] E.A. Macedo, P. Skovborg, P. Rasmussen, Calculation of Phase-Equilibria for Solutions of Strong Electrolytes in Solvent Water Mixtures, Chemical Engineering Science, 45 (1990) 875-882.

[20] E. Waisman, J.L. Lebowitz, Mean spherical model integral equation for charged hard spheres I. Method of solution, The Journal of Chemical Physics, 56 (1972) 3086.

[21] L. Blum, Mean spherical model for asymmetric electrolytes: I. Method of solution, Mol Phys, 30 (1975) 1529-1535.

[22] W.G. Chapman, G. Jackson, K.E. Gubbins, Phase-Equilibria of Associating Fluids Chain Molecules with Multiple Bonding Sites, Mol Phys, 65 (1988) 1057-1079.

[23] W.G. Chapman, K.E. Gubbins, G. Jackson, M. Radosz, Saft - Equation-of-State Solution Model for Associating Fluids, Fluid Phase Equilibr, 52 (1989) 31-38.

[24] W.G. Chapman, K.E. Gubbins, G. Jackson, M. Radosz, New Reference Equation of State for Associating Liquids, Ind Eng Chem Res, 29 (1990) 1709-1721.

[25] M. Wertheim, Fluids with highly directional attractive forces. II. Thermodynamic perturbation theory and integral equations, Journal of Statistical Physics, 35 (1984) $35-47$.

[26] M. Wertheim, Fluids with highly directional attractive forces. IV. Equilibrium polymerization, Journal of statistical physics, 42 (1986) 477-492.

[27] M. Wertheim, Fluids with highly directional attractive forces. III. Multiple attraction sites, Journal of Statistical Physics, 42 (1986) 459-476.

[28] C. McCabe, A. Galindo, SAFT associating fluids and fluid mixtures, Applied Thermodynamics of Fluids, (2010) 215-279.

[29] E.A. Muller, K.E. Gubbins, Molecular-based equations of state for associating fluids: A review of SAFT and related approaches, Ind Eng Chem Res, 40 (2001) 2193-2211. 
[30] I.G. Economou, Statistical associating fluid theory: A successful model for the calculation of thermodynamic and phase equilibrium properties of complex fluid mixtures, Ind Eng Chem Res, 41 (2002) 953-962.

[31] S.P. Tan, H. Adidharma, M. Radosz, Recent advances and applications of statistical associating fluid theory, Ind Eng Chem Res, 47 (2008) 8063-8082.

[32] A. Galindo, A. Gil-Villegas, G. Jackson, A.N. Burgess, SAFT-VRE: Phase behavior of electrolyte solutions with the statistical associating fluid theory for potentials of variable range, The Journal of Physical Chemistry B, 103 (1999) $10272-10281$.

[33] L.F. Cameretti, G. Sadowski, J.M. Mollerup, Modeling of aqueous electrolyte solutions with perturbed-chain statistical associated fluid theory, Ind Eng Chem Res, 44 (2005) 3355-3362.

[34] A. GilVillegas, A. Galindo, P.J. Whitehead, S.J. Mills, G. Jackson, A.N. Burgess, Statistical associating fluid theory for chain molecules with attractive potentials of variable range, J Chem Phys, 106 (1997) 4168-4186.

[35] S.P. Tan, H. Adidharma, M. Radosz, Statistical associating fluid theory coupled with restricted primitive model to represent aqueous strong electrolytes, Ind Eng Chem Res, 44 (2005) 4442-4452.

[36] J. Gross, G. Sadowski, Application of the Perturbed-Chain SAFT Equation of State to Associating Systems, Ind Eng Chem Res, 41 (2002) 5510-5515.

[37] J. Rozmus, J.C. de Hemptinne, A. Galindo, S. Dufal, P. Mougin, Modeling of Strong Electrolytes with ePPC-SAFT up to High Temperatures, Ind Eng Chem Res, 52 (2013) 9979-9994.

[38] B.H. Patel, P. Paricaud, A. Galindo, G.C. Maitland, Prediction of the Salting-Out Effect of Strong Electrolytes on Water + Alkane Solutions, Ind Eng Chem Res, 42 (2003) 3809-3823.

[39] J. Gross, G. Sadowski, Perturbed-chain SAFT: An equation of state based on a perturbation theory for chain molecules, Ind Eng Chem Res, 40 (2001) 1244-1260.

[40] M. Uematsu, E.U. Franck, Static Dielectric-Constant of Water and Steam, J Phys Chem Ref Data, 9 (1980) 1291-1306.

[41] L. Blum, D. Wei, Analytical solution of the mean spherical approximation for an arbitrary mixture of ions in a dipolar solvent, The Journal of Chemical physics, 87 (1987) 555.

[42] H. Zhao, M.C. dos Ramos, C. McCabe, Development of an equation of state for electrolyte solutions by combining the statistical associating fluid theory and the mean spherical approximation for the nonprimitive model, The Journal of Chemical physics, 126 (2007) 244503.

[43] G. Das, S. Hlushak, M.C. dos Ramos, C. McCabe, Predicting the thermodynamic properties and dielectric behavior of electrolyte solutions using the SAFT-VR+DE equation of state, Aiche J, 61 (2015) 3053-3072.

[44] D.Q. Wei, L. Blum, The Mean Spherical Approximation for an Arbitrary Mixture of Ions in a Dipolar Solvent - Approximate Solution, Pair Correlation-Functions, and Thermodynamics, J Chem Phys, 87 (1987) 2999-3007.

[45] J.S. Rowlinson, F.L. Swinton, Liquids and Liquid Mixtures, 3rd ed., Butterworth Scientific, London, 1982. 
[46] E.K. Karakatsani, G.M. Kontogeorgis, I.G. Economou, Evaluation of the truncated perturbed chain-polar statistical associating fluid theory for complex mixture fluid phase equilibria, Ind Eng Chem Res, 45 (2006) 6063-6074.

[47] E.K. Karakatsani, T. Spyriouni, I.G. Economou, Extended statistical associating fluid theory (SAFT) equations of state for dipolar fluids, Aiche J, 51 (2005) 23282342.

[48] T.W. Leland, J.S. Rowlinson, G.A. Sather, Statistical thermodynamics of mixtures of molecules of different sizes, Transactions of the Faraday Society, 64 (1968) 1447-1460.

[49] G. Das, S. Hlushak, M.C. dos Ramos, C. McCabe, Predicting the thermodynamic properties and dielectric behavior of electrolyte solutions using the SAFT-VR+DE equation of state, AIChE Journal, in press (2015).

[50] M. Golovko, I. Protsykevich, Analytic solution of the mean spherical approximation for ion-dipole model in a neutralizing background, Journal of Statistical Physics, 54 (1989) 707-733.

[51] H.G. Zhao, C. McCabe, Phase behavior of dipolar fluids from a modified statistical associating fluid theory for potentials of variable range, J Chem Phys, 125 (2006).

[52] G.J. W. G. Chapman, and K. E. Gubbins, Mol. Phys, 65 (1988).

[53] C.G. Gray, Y.S. Sainger, C.G. Joslin, P.T. Cummings, S. Goldman, ComputerSimulation of Dipolar Fluids - Dependence of the Dielectric-Constant on System Size - a Comparative-Study of Ewald Sum and Reaction Field Approaches, J Chem Phys, 85 (1986) 1502-1504.

[54] J.A. Barker, R.O. Watts, Monte-Carlo Studies of Dielectric Properties of WaterLike Models, Mol Phys, 26 (1973) 789-792.

[55] P.T. Cummings, I. Nezbeda, W.R. Smith, G. Morriss, Monte-Carlo Simulation Results for the Full Pair Correlation-Function of the Hard Dumbbell Fluid, Mol Phys, 43 (1981) 1471-1475.

[56] A. Gil-Villegas, G. Jackson, S.C. McGrother, Computer simulation of dipolar liquid crystals., J Mol Liq, 76 (1998) 171-181.

[57] A.L. Benavides, Y. Guevara, F. Delrio, Vapor-Liquid-Equilibrium of a Multipolar Square-Well Fluid .1. Effect of Multipolar Strengths, Physica A, 202 (1994) 420437.

[58] M. Neumann, O. Steinhauser, G.S. Pawley, Consistent Calculation of the Static and Frequency-Dependent Dielectric-Constant in Computer-Simulations, Mol Phys, 52 (1984) 97-113.

[59] P.T. Cummings, H.D. Cochran, J.M. Simonson, R.E. Mesmer, S. Karaborni, Simulation of Supercritical Water and of Supercritical Aqueous-Solutions, J Chem Phys, 94 (1991) 5606-5621.

[60] A.V. Bandura, S.N. Lvov, D.D. Macdonald, Thermodynamics of ion solvation in dipolar solvent using Monte Carlo mean reaction field simulation, J Chem Soc Faraday T, 94 (1998) 1063-1072.

[61] D.M. Tsangaris, J.J. Depablo, Bond-Bias Simulation of Phase-Equilibria for Strongly Associating Fluids, J Chem Phys, 101 (1994) 1477-1489.

[62] D.P. Visco, D.A. Kofke, Modeling the Monte Carlo simulation of associating fluids, J Chem Phys, 110 (1999) 5493-5502. 
[63] B. Chen, J.J. Potoff, J.I. Siepmann, Adiabatic nuclear and electronic sampling Monte Carlo simulations in the Gibbs ensemble: Application to polarizable force fields for water, J Phys Chem B, 104 (2000) 2378-2390.

[64] D.P. Visco, D.A. Kofke, A comparison of molecular-based models to determine vapor-liquid phase coexistence in hydrogen fluoride, Fluid Phase Equilibr, 158 (1999) 37-47.

[65] B. Chen, J.I. Siepmann, A novel Monte Carlo algorithm for simulating strongly associating fluids: Applications to water, hydrogen fluoride, and acetic acid, J Phys Chem B, 104 (2000) 8725-8734.

[66] B. Chen, J.I. Siepmann, Partitioning of alkane and alcohol solutes between water and (Dry or wet) 1-Octanol, J Am Chem Soc, 122 (2000) 6464-6467.

[67] N.A. Busch, M.S. Wertheim, Y.C. Chiew, M.L. Yarmush, A Monte-Carlo Method for Simulating Associating Fluids, J Chem Phys, 101 (1994) 3147-3156.

[68] H. Docherty, A. Galindo, A study of Wertheim's thermodynamic perturbation theory (TPT1) for associating fluids with dispersive interactions: the importance of the association range, Mol Phys, 104 (2006) 3551-3560.

[69] S.J. Han, H.Q. Pan, Thermodynamics of the Sodium Bromide-Methanol-Water and Sodium Bromide-Ethanol-Water 2 Ternary-Systems by the Measurements of Electromotive-Force at 298.15-K, Fluid Phase Equilibr, 83 (1993) 261-270.

[70] B. Maribo-Mogensen, G.M. Kontogeorgis, K. Thomsen, Modeling of Dielectric Properties of Aqueous Salt Solutions with an Equation of State, J Phys Chem B, 117 (2013) 10523-10533. 